\title{
Pronóstico a largo plazo de la neumonía adquirida en la comunidad en mayores de 60
}

Prognosis After Community-Acquired Pneumonia in the Elderly. A Population-Based 12-Year Follow-up Study. Koivula I; Stén M; Makela PH; et al. Arch Intern Med. 1999;159:1550-1555.

\section{Objetivo}

Comparar la sobrevida a largo plazo, de las personas que padecieron un episodio de neumonía aguda de la comunidad (NAC) en relación con las que no la padecieron.

\section{Diseño}

Cohorte prospectiva observacional*.

\section{Lugar}

Finlandia.

\section{Pacientes}

De una población de 4167 personas de 60 años o más ( $99 \%$ del total de la población anciana del lugar), 122 de ellos sobrevivieron a una NAC durante el período comprendido entre 1983 y 1985. Se realizó seguimiento prospectivo, de estas personas, desde 1983 a 1994 con una media de seguimiento de 9.2 años. Las causas de muerte fueron obtenidas del registro de los certificados de defunción.

Evaluación de Factores Pronósticos

Hipertensión; diabetes; enfermedad coronaria; insuficiencia cardiaca; enfermedades pulmonares, renales, endocrinas, neoplásicas; alcoholismo; internaciones e inmunizaciones previas.

\section{Medición de los Resultados Principales}

Riesgo Relativo (RR) de muerte en pacientes que sobrevivieron a una NAC comparado con el de los habitantes de esa misma región y de la misma edad sin NAC. El RR fue calculado para muerte relacionada a neumonía, muerte por enfermedad cardiovascular y mortalidad total.

\section{Resultados}

El tiempo de sobrevida a largo plazo fue significativamente menor en personas que habían tenido NAC o NAC por Streptococcus pneumoniae (NACN) que en el resto de la población en estudio. La sobrevida de los 122 pacientes que padecieron una NAC entre 1983 y 1985, fue dè $89 \%$ al año del primer episodio, $80 \%$ a los 2 años, $60 \%$ a los 5 años y $39 \%$ después de 10 años. En cambio, la sobrevida de las personas que no tuvieron NAC fue de $96 \%$ al año de seguimiento, $92 \%$ a los 2 años, $81 \%$ a los 5 años y $61 \%$ después de 10 años.

\begin{tabular}{|c|c|c|c|c|c|}
\hline & $\begin{array}{l}\text { Mortalidad relac. a neumonía } \\
\text { RR (IC } 95 \%)\end{array}$ & $\begin{array}{l}\text { Mortalidad T } \\
\text { RR (IC 95\%) }\end{array}$ & & $\begin{array}{l}\text { Mortalidad c } \\
\text { RR (IC } 95 \% \text { ) }\end{array}$ & vascular \\
\hline NAC & $2.1(1.3-3.4)$ & $1.5(1.2-1.9)$ & $p=0.001$ & $1.4(1.0-1.9)$ & $p=0.02$ \\
\hline NACN & $2.8(1.5-5.3)$ & $1.6(1.1-2.2)$ & $p=0.01$ & $1.6(1.0-2.4)$ & $p=0.04$ \\
\hline
\end{tabular}

Los factores pronósticos que predijeron mortalidad en este grupo en forma significativa fueron: el antecedente de haber padecido una NAC o NACN; edad $>70$ años; sexo masculino; cardiopatía; diabetes e internaciones previas.

\section{Conclusiones:}

Los pacientes ancianos tratados por NAC tienen mayor riesgo de mortalidad en los años subsiguientes.

La mortalidad relacionada a neumonía, fue dos veces mayor en pacientes que sobrevivieron a una NAC y tres veces mayor en los que sobrevivieron a una NACN que la de la población anciana de la región. Teniendo en cuenta la alta incidencia y el mal pronostico de la neumonía a largo plazo, podría concluirse en la necesidad de dirigir los esfuerzos para realizar la prevención del primer episodio con vacunación anti-influenza y antineumococcica.

Fuente de Financiamiento: Este estudio fue financiado por: Paulo Foundation, Päivikki and Sakari Sohlberg Foundation, Tampere Tuberculosis Foundation, Väinö and Laina Kivi Foundation y Orion Corporation Research Fòundation.

\section{COMENTARIO}

La neumonía sigue siendo una de las causas principales de muerte en todo el mundo.

El Streptococcus pneumoniae es el agente causal más frecuente de NAC en pacientes mayores de 50 años.

Según datos estadísticos de Estados Unidos y Finlandia, la neumonía y la gripe están dentro de las cinco primeras causas de muerte en la población mayor de 65 años.

Algunas enfermedades crónicas, especialmente las pulmonares y cardiovasculares, han sido reconocidas como factores predisponentes para la neumonía ${ }^{1}$

La enfermedad pulmonar obstructiva crónica, frecuente en el anciano, se asocia a un alto riesgo de neumonía. Las enfermedades cardiovasculares tienen un gran impacto en la salud pública, no sólo por su frecuencia en esta población, sino porque aumentan al doble el riesgo de contraer neumonía y el riesgo de mortalidad asociada. Enfermedades crónicas han sido observadas en el 23 al $92 \%$ de los pacientes adultos con NAC internados y en el 6 al $56 \%$ de los pacientes tratados en forma ambulatoria.

Debido a esto surge, como estrategia racional, dirigir todos los esfuerzos para realizar la prevención de la neumonía en la población de alto riesgo mediante la inmunización, en forma anual (en otoño), con vacuna anti-influenza y una sola dosis de antineumocóccica (la revacunación, con ésta última, en la población anciana no es una recomendación formal) $)^{2-4}$

*Ver glosario

Dra. Liliana Stern

Sección Infectología. Hospital Italiano de Buenos Aires.

\section{Referencias}

1-Korvula I, Sten M, Mäkelä PH. Risk Factors for Pneumonia in the Elderly. Am J Med 1994; 96: 313-320.

2-0̈rtavist $\ddot{A}$, Hedlund J, Burman L, Elbel E, et al. Randomised trial of 23-valent pneumococcal capsular polysacharide vaccine in prevention of pneumonia in middle-age and elderly people. Lancet 1998:351:399-403.

3-Nichol LK, Margolis KL, Wuorenma J, von Sternberg T. The efficacy and cost effectiveness of vaccination against influenza among elderly persons living in the community. N Engl I med. 1994; 12: $778-784$.

4-Butler JC, Breiman RF, Campbell JF, Lipman HB, Broome CV, Facklam RR. Pneumococcal Polysaccharide Vaccine Efficacy, An Evaluation of Current Recommendations. JAMA 1993; 270: 1826-1831. 